\title{
A New Approach to Bridge Infrastructure Management
}

\author{
Maria Rashidi ${ }^{\mathrm{a}^{*}}$ \\ Peter Gibson ${ }^{\mathrm{b}}$ \\ Tin Kin $\mathrm{Ho}^{\mathrm{a}}$
}

\begin{abstract}
The maintenance of bridges as a key element in transportation infrastructure has become a major concern due to increasing traffic volumes, deterioration of existing bridges and well-publicised bridge failures. The main goal of this study is to develop a requirement-driven decision support methodology for remediation of concrete bridges within acceptable limits of safety, serviceability and sustainability. The proposed model includes two phases: Phase one is focused on condition assessment and priority ranking of bridge projects which makes use of an integrated priority index addressing a variety of factors. Phase two includes a multi criteria decision making technique which is able to select the best remediation strategy at both project and network level. The modified Simple Multi Attribute Rating Technique (SMART) is used as a decision analysis tool that employs the eigenvector approach of the Analytical Hierarchy Process (AHP) for criteria weighting.
\end{abstract}

\section{Key words: Bridge management; Decision Support System (DSS); Simple Multi Attribute Rating Technique (SMART); Analytical Hierarchy Process (AHP); Maintenance; Rehabilitation and Replacement (MR\&R).}

\section{Introduction}

Bridges are often subjected to high loads, harsh environments, and accidental damage. Determining what level of repair is required to achieve the most economical lifespan from a bridge structure has been a source of dilemma for asset managers and owners for many years. There are approximately 2.5 million bridges on the global higher transportation network. A recent study on bridge inventory estimated that there are approximately 50,000 bridges in Australia and only approximately $18 \%$ were constructed after 1976 . Due to changes and increases in traffic load, structural degradation, and design code, many of these bridges do not meet the current Australian standards ${ }^{1}$. In 2005, the US Federal Highway Agency (FHWA) stated that $28 \%$ of their bridges are rated deficiently. In Europe this figure varies by around $10 \%$. Nevertheless, if we consider a rough average of 20\% deficiency, almost 500,000 bridges require remediation and improvement ${ }^{2}$.

${ }^{a}$ SMART Infrastructure Facility, University of Wollongong, 2522, Australia

${ }^{\mathrm{b}}$ School of Mechanical, Materials, Mechatronics, University of Wollongong, 2522, Australia

*Corresponding author: Maria Rashidi mrashidi@uow.edu.au http://dx.doi.org/10.14453/isngi2013.proc.38 
In accordance with the limited funding for bridge management, maintenance, rehabilitation and replacement (MR\&R) strategies have to be prioritised. A conservative bridge assessment will result in unnecessary actions, such as costly bridge strengthening or repairs ${ }^{3}$. But on the other hand, any bridge maintenance negligence and delayed actions (or ignoring the cause of defects) may lead to heavy future costs or degraded assets.

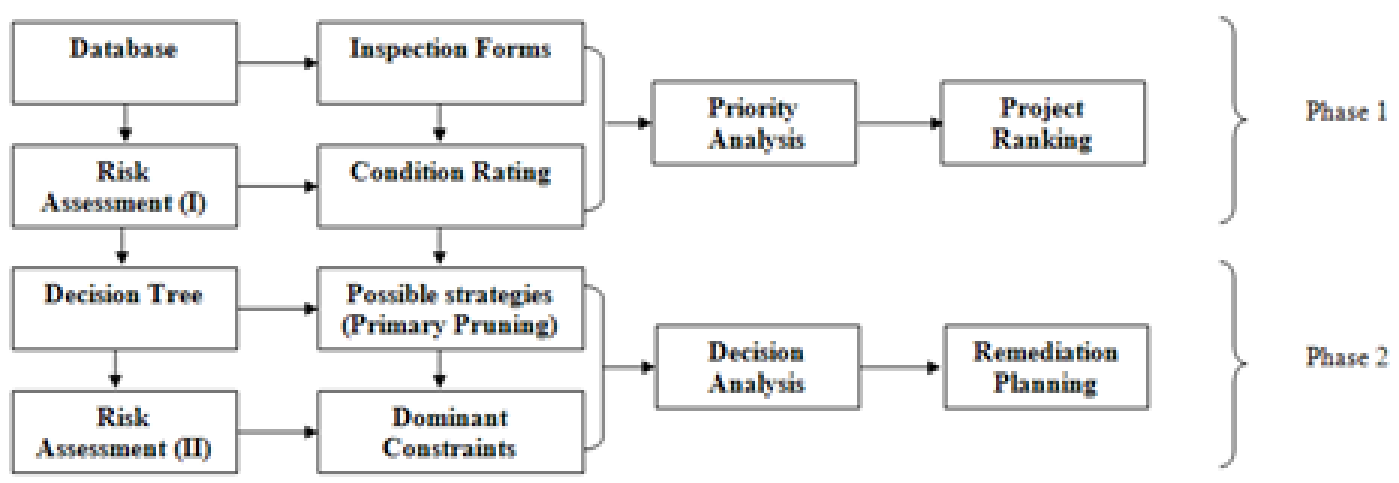

Figure 1. Conceptual Framework for Bridge Management ${ }^{4}$

\section{The Proposed Framework for Bridge Infrastructure Management}

The system methodology presented in this paper deals with the development of a knowledgebased decision support model for bridge infrastructure management as a solution for the problems and limitations of the existing models. The proposed model is expected to be flexible and capable of handling multi-layer of data and dealing with multi-objective nature of the decision. The working model includes a procedure for condition assessment in order to prioritise bridges in a network for any necessary intervention and finally proposing a remediation strategy at both project level and network level. Classifying all the possible actions (including MR\&R strategies and/or treatment options), finding the main constraints and finally employing a suitable decision analysis tool are the main components of the proposed system. Figure 1 shows the overall working framework including two main phases which will finally lead to two major outputs: 1) Project Ranking and 2) Remediation Planning.

Multi criteria nature of the decision making involves various parameters with different importance level. Weighting the engaged factors has been partially accomplished through expert judgements employing Analytical Hierarchy Process (AHP) as a strong tool designed for this purpose. Through the AHP, decision problems are decomposed into a hierarchical structure, and both qualitative and quantitative information can be used to derive ratio scales between the decision elements at each hierarchical level by means of pair wise comparisons. With comparative judgments, users are requested to set up a comparison matrix at each hierarchy by comparing pairs of criteria or sub-criteria. A scale of values ranging from 1 (indifference) to 9 (extreme preference) is used to express the users preference. Finally, in the synthesis of priority stage, each comparison matrix is then solved by an eigenvector method for determining the criteria importance ${ }^{5}$. 


\section{Phase One: Project Ranking}

The reliability of decisions to prioritise bridges for fund allocation is highly dependent upon the thoroughness of the condition assessment and diagnosis process ${ }^{4}$. Most of the existing approaches are commonly based on subjective structural condition assessment. Parameters such as functionality and client preferences may not be specifically addressed in them. As a result, one of the main objectives of this research was to propose an integrated index for the bridge rating, in a requirement driven context. The developing condition rating method described in this paper is an important step toward this aim and along with adding more holism and objectivity to the current methods. The analysis and quantification of Structural Efficiency (SE), Functional Efficiency (FE) and Client Impact Factor (CIF) are addressed in the proposed model.

The first step to evaluate structural efficiency is dividing the bridge into elements generally made of a similar material. The inspector estimates and records the quantities of the bridge element in each condition state independently. The total quantity must be measured in the correct units for the elements. The element condition index can be calculated as the current value divided by the initial value of the bridge element. To describe the overall condition status of structural elements, the Element Structural Condition Index (ESCI) is introduced as:

$$
E S C I=\frac{\Sigma(q i \times c i)}{\Sigma q i}
$$

- $q i$ is the quantity of elements reported in condition index $c i$

- $c i$ is the condition of sub-element $i \quad c i \in(1,2,3,4)$

According to Equation 1, the element condition index ranges from 1 to 4 . In order to be in harmony with the existing evaluation, the quantities assigned for the relative evaluation of the involved parameters (achieved through expert judgements) have been limited to the same range (see Table 1).

Generally, the prevailing condition (rating) of the particular element may cause some inaccuracies in the overall structural assessment. For example, a minor component with worse condition may unreasonably raise the rating value of element under which the component is grouped. This problem has been resolved with the introduction of an element structural significance factor $(\mathrm{Si})$ which is not dependent on the prevailing condition of components (see Table 1). The higher numbers represent the superior importance of structurally critical members which have a great impact on the strength and safety of the structure and where failure of the member could lead to catastrophic collapse.

Different materials have different contributions to the structural efficiency of a bridge. For example reinforced concrete is more vulnerable than steel and the structural vulnerability of precast concrete is more than reinforced concrete. Therefore material factor should be considered in the structural assessment of bridge elements. Table 1 presents the vulnerability factor of common materials used in concrete bridges introduced as Mi which is obtained from the work of Valenzuela et al. $(2010)^{6}$ and validated by the judgements of structural engineers. Based on vulnerability of different materials it varies between 1 and $4^{7}$. 
Bridge elements deteriorate over an extended period of time and the rate of deterioration is a function of various parameters. Apart from some pre-existing factors such as design and construction, there are several post existing causes involved in the structural efficiency of bridges. These include the environment where the structure is located in, the length of time the structure has been in service (Age), the function the structure is required to perform (Road Class) and the quality of inspection and monitoring. The impact of $\mathrm{CF}$ on the bridge structural efficiency can be evaluated through Equation 2. The weights of the involved parameters have been estimated using AHP and the associated ratings are defined based on the classifications presented in Table 1.7

$$
C F=0.411 A+0.120 E+0.107 R+0.362 I
$$

The overall Structural Efficiency index (SE) is a dimensionless relative parameter that integrates all the elements which influence structural effectiveness and is estimated as follows ${ }^{8}$ :

$$
\mathrm{SE}=\frac{\mathrm{CF} 2(\mathrm{Mi} \times \mathrm{SI} \times \mathrm{ESCI} 1)}{16 \mathrm{n}}
$$

The range of SE varies from 1 to 4 . The priority for remedial action increases as the number increases (n represents the number of element types). The modern BMS considers the quality of service (functional efficiency) in addition to structural efficiency. Yanev (2007) ${ }^{9}$ stated that "the functional life of bridges is less than the structural life," e.g., 25 to 50 years (in high traffic growth), compared to 50 to 100 years (except disasters).

According to Rashidi and Lemass $(2011)^{4}$, the bridge functional efficiency is dependent on the traffic volume that it can withstand, which is mainly related to the load bearing capacity of the bridge, existing number of lanes or the width of the deck, vertical clearance and the barriers. The drainage system, provisions for pedestrians and cyclists and any post design changes should also be carefully considered in the assessment process. Any deficiency associated with the above items can reduce the level of service and accelerate the deterioration process. For this reason, it is advantageous to consider the elimination of these deficiencies within the decision making process. Five main deficiencies that can seriously affect bridge safety and serviceability are: load bearing capacity, vertical clearance, width, barriers and the drainage system. The overall functional efficiency factor (FE) can be calculated using the ratings (See Table 1) and the weights as shown in the Equation 4.

$$
F E=0.7 L c+0.1 V c+0.1 W b+0.05 B b+0.05 D s
$$

- Lc is the load bearing capacity

- Vc is the vertical clearance

- Wb is the width

- $\mathrm{Bb}$ is the barrier

- Ds is the drainage system

The nature of a bridge site and the extent of the bridge remediation treatment may cause decision makers to close bridge lanes or create alternative routes or bypasses to control the traffic flow. Excessive traffic delay times often result in negative feedback from both the road users and 
their political representatives. Client Impact Factor (CIF) helps build the social implications of remediation into the risk assessment process. It is a vast improvement on the 'do nothing' course of action. On the other hand, the bridge's importance for economic activity can accelerate the decision making process toward 'replacement' or 'rehabilitation' 10 . This factor can be ranked based on the level of bridge criticality in terms of socio-economic, political and historical considerations as shown in Table 1. The key decision maker or bridge maintenance planner will be responsible to rate this parameter based on their understanding of client preferences. Finally the Priority Index (PI) integrates all the above mentioned factors that will influence decision making through the following equation:

$$
\mathrm{PI}=0.6 \mathrm{SE}+0.2 \mathrm{FE}+0.2 \mathrm{CIF}
$$

Table 1. Ratings of All the Contributed Parameters in Condition Rating and Prioritisation

\begin{tabular}{|c|c|c|c|c|c|c|c|c|c|c|c|c|}
\hline \multirow{3}{*}{ Ratings } & \multicolumn{6}{|c|}{ STRUCTURAL EFFICIENCY (SE) } & \multirow{2}{*}{\multicolumn{5}{|c|}{ FUNCTIONAL EFFICIENCY (FE) }} & \multirow{3}{*}{$\begin{array}{l}\text { CLIENT IMPACT } \\
\text { FACTOR } \\
\text { (CIF) }\end{array}$} \\
\hline & \multirow{2}{*}{$\begin{array}{l}\text { Structural Significance Factor } \\
\text { (Si) }\end{array}$} & \multirow{2}{*}{$\begin{array}{c}\text { Material } \\
\text { Vulnerability Factor } \\
\text { (Mi) }\end{array}$} & \multicolumn{4}{|c|}{$\begin{array}{l}\text { Causal Factors } \\
\text { (CF) }\end{array}$} & & & & & & \\
\hline & & & $\begin{array}{l}\text { Age } \\
\text { (A) }\end{array}$ & $\begin{array}{c}\text { Environmental } \\
\text { Aggresive } \\
\text { Factor (E) }\end{array}$ & $\begin{array}{l}\text { Road Class } \\
\quad(R)\end{array}$ & $\begin{array}{l}\text { Inspection } \\
\text { quality } \\
\text { (I) }\end{array}$ & $\begin{array}{l}\text { Load Bearing } \\
\text { Capacity (Lc) }\end{array}$ & $\begin{array}{c}\text { Vertical } \\
\text { Clearance }\left(V_{0}\right)\end{array}$ & $\begin{array}{l}\text { Width } \\
\text { (Wb) }\end{array}$ & $\begin{array}{c}\text { Bridge Barrier } \\
\text { (Bb) }\end{array}$ & $\begin{array}{c}\text { Drainage } \\
\text { system (Ds) }\end{array}$ & \\
\hline 1 & Barriers, Footway, Kerbs, Joints & Steel & Recently Built & Low & Minor & Very High & $L c \geq 1$ & Vc $\leq 5$ & $\mathrm{~Wb} \leq 5$ & $B b \leq 5$ & Very Good & Low \\
\hline 2 & Foundation, Abutment, Wingwalls & Reinforced Concrete & New & Medium & Local Access & High & $0.9 \leq \mathrm{L} c<1.0$ & $5<\mathrm{V} c \leq 12$ & $5<W b \leq 12$ & $5<B b \leq 12$ & Good & Medium \\
\hline 3 & Deck, Bearings & Precast Concrete & Old & High & Collectors & Medium & $0.7 \leq L \mathrm{~L} c 0.9$ & $12<V c \leq 20$ & $12<W b \leq 20$ & $12<B b \leq 20$ & Fair & High \\
\hline 4 & Beams, Headstocks, Piers & Prestressed Concrete & Very Old & Very High & Arterials & Low & Lc<0.7 & $V_{c} \geq 20$ & $W b \geq 20$ & $\mathrm{Bb} \geq 20$ & Poor & Very High \\
\hline
\end{tabular}

Using PI enables bridge/funding agencies to make decisions and set objectives backed up by strong logic. By using this technique all bridges are sorted in descending order starting with the bridge with the highest ranking index, the required actions are carried out until the allocated funds are exhausted.

\section{Phase Two: Remediation Planning}

Sound decision making requires including multiple and conflicting criteria in the process. Five major categories of criteria including safety, functionality, sustainability, environment and legal/political constraints have been identified through level two of risk assessment. Different decision analysis tools have also been analysed and the modified Simple Multi Attribute Ranking Method (SMART) was selected as the main frame work for strategy selection.

Through the SMART process, firstly, the problem under consideration is mapped into a hierarchy, including at least three main levels: goal, criteria and alternatives. The decision subcriteria might be general and they may therefore require to be broken down into more specific sub-criteria introduced as attributes in an extra level of hierarchy. Each criterion has a weight indicating its importance and reflecting the organizational policy. These weights are defined by the decision makers employing the pair wise comparison approach embedded in the AHP and will vary for different projects with different decision makers ${ }^{10}$. 
The AHP has the major benefit of allowing the decision makers to carry out a consistency check for the developed judgment in regard to its relative importance among the decision making components. Therefore, the decision maker(s) can modify their judgments to improve the consistency and to supply more-informed judgments under consideration. The procedure is also able to provide flexibility in selecting the criteria to be used to evaluate the rehabilitation strategies and even increasing or decreasing the numbers of levels (associated with the criteria) in the hierarchy.

\section{Conclusion}

The main scope of this research was to develop a decision support methodology for bridge remediation that would improve knowledge in the area of infrastructure management. Based on the achieved developments, this research made a number of contributions which will be beneficial to transportation agencies and infrastructure asset managers. The proposed model is able to add more objectivity to the existing systems through quantifying the major parameters and considering both the project and network aspects of the infrastructure management plan. The analysis of case studies and the feedback received from the experts confirms the applicability of the system.

\section{References}

${ }^{1}$ Sumitomo, Bridge repair market statistics, 2009, URL: http://www.iceng.com.au/files/link/Bridge-market-statistics.pdf.

${ }^{2}$ Freudenthaler. B, Gutenbrunner.G, Stumptner. R, \& Kung. J., "Case-based decision support for bridge monitoring", Proceeding of the third international Multi-Conference on Computing in the global information technology, 2008, pp. 31-36.

${ }^{3}$ Stewart, M. G., "Reliability-based assessment of ageing bridges using risk ranking and life cycle cost decision analyses", Reliability Engineering \& System Safety, Vol. 74, No. 3, 2008, pp. 263-273. http://dx.doi.org/10.1016/S0951-8320(01)00079-5

${ }^{4}$ Rashidi, M. and Lemass B., "Holistic decision support for bridge remediation", The 4th International Conference on Construction Engineering and Project Managment (ICCEPM), 2011, Sydney, Australia.

${ }^{5}$ Bello-Dambatta, A., Farmani R., Javadi A. A. and Evans B. M., "The Analytical Hierarchy Process for contaminated land management", Advanced Engineering Informatics, Vol. 23, No. 4, 2009, pp. 433-441. http://dx.doi.org/10.1016/j.aei.2009.06.006

${ }^{6}$ Valenzuela, S., de Solminihac H. and Echaveguren T., "Proposal of an integrated index for prioritization of bridge maintenance", Journal of Bridge Engineering, Vol. 15 , No. 3, 2010, pp. 337-343. http://dx.doi.org/10.1061/(ASCE)BE.1943-5592.0000068

${ }^{7}$ Rashidi, M. and Gibson P., "Proposal of a methodology for bridge condition assessment", Australasian Transport Research Forum (ATRF), 2011, Adelaide, Australia.

${ }^{8}$ Rashidi, M. and Gibson P., "A methodology for bridge condition evaluation", Journal of Civil Engineering and Architecture, Vol. 6, No. 9, 2012, pp. 1149-1157.

${ }^{9}$ Yanev, B., Bridge Management, John Wiley \& Sons, Hoboken, New Jersey, 2007. http://dx.doi.org/10.1002/9780470168059

${ }^{10}$ Rashidi, M. and Lemass B., "A Decision Support Methodology for Remediation Planning of Concrete Bridges", Journal of Construction Engineering and Project Management (JCEPM), Vol. 1, No. 2, 2011, pp. 1-10. http://dx.doi.org/10.6106/JCEPM.2011.1.2.001 